\title{
ESTABILIDAD Y CONVERGENCIA EN EL MODELO CLÁSICO DE CRECIMIENTO DE LA POBLACIÓN
}

\author{
José Carlos Ramírez*
}

Departamento de Economía, Tecnológico de Monterrey, Campus Cindad de México

\author{
José B. Morelos \\ CEDDU, El Colegio de México, A. C.
}

(Recibido 23 de septiembre 2001, aceptado 15 de noviembre 2002)

\section{Resumen}

Este trabajo propone un nuevo enfoque para analizar el modelo clásico de crecimiento de la población. Se parte de la ecuación de Verhulst para modelar el principio de población de Malthus y, mediante la introducción de nuevos parámetros ofrece una explicación de las condiciones de estabilidad y convergencia de las trayectorias de crecimiento basadas en el modelo de Ricardo. La idea central de esta propuesta es que, contrario al punto de vista convencional, Malthus y Ricardo ofrecen el primer modelo agregado en el que se observa. explícitamente las relaciones entre las variables económicas y demográficas. Los modelos recientes de crecimiento económicos son, de hecho, herederos de este enfoque y sus diferencias estriban en las distintas formas en que cada uno de ellos incorpora el principio de población de Malthus en sus ecuaciones.

\begin{abstract}
This paper proposes a new approach to analyze the classical model of population growth. By using the Verhulst equation we model Malthus' population principle and state the stability and convergence conditions of Ricardo's model of economic growth. Our point of view is that Malthus and Ricardo developed the first aggregate model in which the economic and demographic variables can be explicitly related. In fact, recent models of economic growth analize that dual relation by incorporating Malthus' population principle into their theoretical frameworks in different fashions.
\end{abstract}

Clasificación JEL: J10, 041, R11

Palabras clave: Tendencias demográficas, Crecimiento económico, Análisis del crecimiento

* Departamento de Economía, Tecnológico de Monterrey, Campus Ciudad de México. Calle del Puente 222, Col. Ejidos de Huipulco, Deleg. Tlalpan México, D.F. Teléfono 52(55)54831328, Correo Electrónico: jcrsanch@itesm.mx

Los autores desean agradecer a dos dictaminadores anónimos sus valiosas observaciones. 


\section{Introducción}

El objetivo de este trabajo es mostrar que los economistas clásicos desarrollaron el primer modelo de crecimiento en el que la dinámica de la población, junto con la ley de rendimientos decrecientes, determinaba la convergencia de la economía al estado estacionario. En particular, nos concentramos en los trabajos de Malthus y Ricardo, por considerar que en ellos se resumen todos los elementos necesarios para entender el crecimiento de la economía en el largo plazo (Eltis 2000).

El principio de población de Malthus es esencial para explicar la relación entre las variables económicas y demográficas en el modelo clásico. La adopción de este principio por Ricardo permitió darle fundamento a la ley de rendimientos decrecientes y, con ello, a todas sus sombrías opiniones sobre el estado estacionario. Sin la inclusión de este principio, no sólo sería ininteligible la concepción de largo plazo de Ricardo sino, también, su teoría del valor y distribución como explicación del crecimiento económico.

La importancia de un tema de esta naturaleza no es de ninguna manera de orden histórico, ya que si ése fuera el caso poco podríamos agregar al enorme caudal de investigaciones ya existentes (véase Birsdall 1993). La idea es mas bien ofrecer una explicación alternativa a la visión generalizada de que la población juega un papel pasivo en los modelos de crecimiento, incluido el clásico. La exposición del modelo clásico es, en este contexto, un medio para resaltar que los resultados macroeconómicos de largo plazo, pronosticados por los diferentes tipos de modelos, son en gran medida, una consecuencia directa de la manera en que éstos incorporan el principio de población de Malthus. La aseveración es válida también para los modelos recientes neoclásicos en los que el principio de población es trasladado de su original ambiente macroeconómico al ámbito de la familia, tal como lo dejamos en claro en otro trabajo (Ramírez y Morelos 2002).

El trabajo consta de dos partes. La primera expone el principio de población, mientras que la segunda analiza la manera en que Ricardo adopta este principio en su modelo de crecimiento. En ambas partes, hacemos uso de la ecuación de Verhulst como un medio expedito para hacer explícitas las modificaciones hechas por Ricardo a la versión original del principio. Las conclusiones incorporan una breve discusión acerca de los resultados alcanzados.

\section{La ecuación de Verhulst y el principio de población malthusiano}

Como es bien sabido, Malthus (1977) escribió su Ensayo sobre el principio de población como una reacción a los juicios optimistas que sus contemporáneos formulaban sobre el progreso ilimitado de la sociedad. ${ }^{1}$ En su Ensayo, Malthus expresó su temor de que la población creciese más rápido que los medios de

1 Para esos contemporáneos, el porvenir de la sociedad humana, la supremacía de la razón, los progresos de la ciencia y la legislación social se conceptuaban como los medios idóneos para superar los egoísmos individuales, dominar los afanes por la ganancia y subordinar 
subsistencia y de que, lejos de tratarse de un problema distante en el tiempo, era un obstáculo que cobraba vigencia en las circunstancias de su época. Para darle validez de ley a sus argumentos, enunció un principio en el que sostuvo que la población crecía geométricamente mientras que los medios de subsistencia lo hacían aritméticamente. El principio buscaba dejar en claro que el crecimiento desmesurado de la población era el factor causal de la pobreza en las clases bajas de la sociedad y, con ello, refutar el optimismo reinante (véase Winch 1997).

La mecánica del sistema que está detrás del principio de población de Malthus puede ser explicada formalmente con la ayuda de la ecuación logística de Verhulst:

$$
\frac{\mathrm{d} P}{\mathrm{~d} t}=a P-b P^{2}
$$

donde $P$ es la población y $a$ y $b$ son constantes no negativas que representan, res pectivamente, las tasas medias de natalidad y mortalidad. De acuerdo con (1), la población crece en forma exponencial según la trayectoria

$$
P(t)=\frac{a P_{0}}{\left(a-b P_{0}\right)}\left[\frac{b P_{0}}{\left(a-b P_{0}\right)}+e^{-a t}\right]^{-1}
$$

la cual es precisamente la solución particular a la ecuación logística que satisface la condición inicial $P(0)=P_{0}\left(\mathrm{y} c_{1} \neq 0\right)$. Los límites al crecimiento de (2) están fijados en la figura 1 por: $\lim _{t \rightarrow \infty} P(t)=a / b$ y por $\lim _{t \rightarrow-\infty} P(t)=0$. Estos límites dan la consabida forma de $S$ a la curva de Verhulst. La constante $a / 2 b$ es establecida como una cota intermedia para señalar la cantidad mínima de individuos que es requerida para garantizar el reemplazo de la población. El nivel de esta cota es variable y depende del tipo de especie bajo consideración.

\section{Figura 1.}

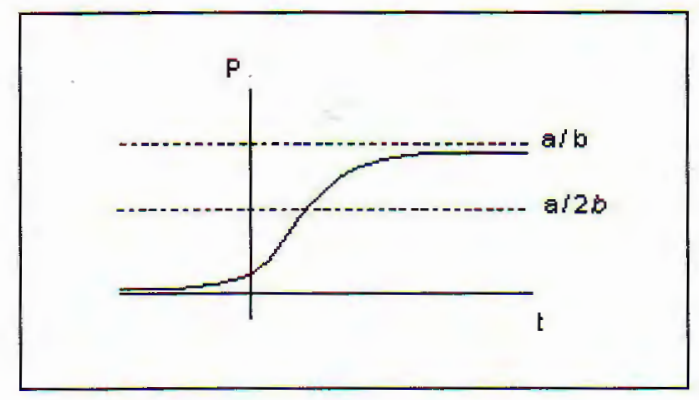

\footnotetext{
los sentidos al espíritu en el asunto de la reproducción humana. Además, la confianza en la omnipotencia de la ciencia para postergar en forma indefinida la muerte y asegurar el suministro de subsistencias durante un futuro lejano eran razones suficientes para que esos autores se despreocuparan en el interim, en la prefiguración de ese mundo feliz, del crecimiento de la población, cualesquiera que éste fuera (Le Bras, 1983).
} 
Para aprovechar estos resultados volvamos a la ecuación (1). El segundo componente a la derecha de esa ecuación, $-b P^{2}$ con $b>0$, es llamado el factor de inhibición o de competencia que, en terminología de Malthus, podemos decir que es producido por los frenos positivos (enfermedades, hambrunas y malnutrición) operantes en caso de un exceso relativo de la población. Estos frenos, junto con los preventivos (reducción voluntaria de la fecundidad), actúan como contrapeso al deseo natural de los hombres por la reproducción de la especie(pasión de los sexos), y se activan al bajar los niveles de subsistencias. ${ }^{2} \mathrm{El}$ primer componente, $a P$ con $a>0$, es, por su parte, el factor de expansión de la población que Malthus supone como una función positiva de los medios de subsistencia, $S$, y como una función negativa de los frenos preventivos. El resultado final de la acción de ambos componentes está determinado por un mecanismo del tipo Lotka-Volterra que se explica por medio del siguiente sistema no lineal de ecuaciones diferenciales.

$$
\begin{aligned}
\frac{\mathrm{d} P}{d t} & =r_{0} P(t)\left[1-\frac{P(t)}{K}\right], \\
\frac{\mathrm{d} S}{\mathrm{~d} t} & =\gamma S, \\
P_{0} & =S_{0}=K .
\end{aligned}
$$

La primera ecuación del sistema (3) no es más que una variante de (1) en la que la población crece a una tasa instantánea $r_{0}=a-b$, acotada por $K$, mientras que la segunda representa el postulado malthusiano de que los medios de subsistencia, $S$, crecen a un tasa aritmética $\gamma$. La pendiente de la curva solución del sistema expresa la razón de cambio de $P$ ante un incremento en $S$, o, dicho de otra manera, indica que el crecimiento exponencial sugerido por Malthus es una función exclusiva de las tasas de mortalidad y natalidad (no hay migración) y, éstas a su vez de los medios de subsistencia. "Population is food controlled", como correctamente señala Keifitz (1983).

El par de soluciones linealmente independientes $(r \neq \gamma)$ obtenidas en (4), tras linealizar el sistema (3), dan como resultado la curva de Verhulst con pendiente en un primer tramo igual a $c_{2} B e^{(\gamma-r) t} / c_{1} A$ (figura 2$) .^{3}$ En efecto, si $c_{1} \neq 0, c_{2} \neq 0$, y $A \neq 0$, la curva crecerá por encima de $K$ en el intervalo en donde $\gamma \geq r \geq 0$. Fuera de ese intervalo la curva experimentará un decrecimiento en su segundo tramo, que la llevará de regreso hasta $K$ en virtud de

2 El aplazamiento de la edad al matrimonio y la recucción de la fecundidad son consecuencia de los frenos preventivos, los cuales funcionan en el análisis de Malthus como restricciones morales. La disminución de la población producida por la miseria opera, por su parte, como un freno positivo al aumentar, sobre todo, la mortalidad de los menores de edad (Le Bras, 1983).

3 La linealización de (3) es posible debido a que el valor del determinante de los coeficientes es diferente de cero (de hecho es positivo e igual a $r \gamma$ ) ya que $\lim _{\left(P_{2} S\right) \rightarrow(0,0)} b P / \sqrt{P^{2}+S^{2}}=0$. 
que el

$$
\begin{aligned}
\lim _{t \rightarrow \infty} \frac{S}{P} & =\frac{c_{2} B e^{(\gamma-r) t}}{c_{1} A}=0=K, \\
P & =c_{1} A e^{r t} \\
S & =c_{2} B e^{\gamma t}
\end{aligned}
$$

Figura 2. Equilibrio Malthusiano.

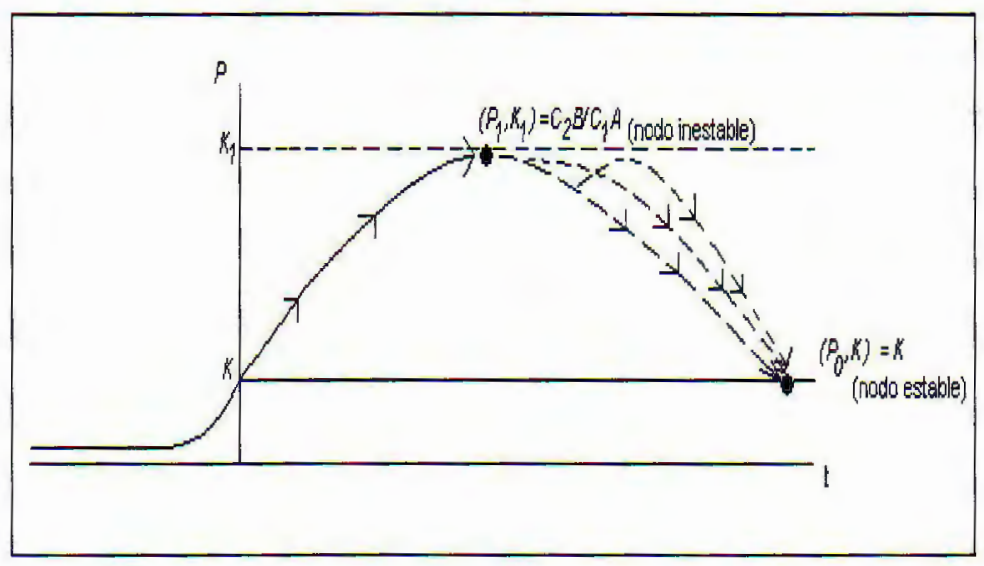

La nueva curva solución tiene una condición inicial y dos supuestos de comportamiento que regulan su crecimiento. La condición inicial $P_{0}=S_{0}=K$ significa que hay un estado del desarrollo en el cual el nivel de ingreso de la sociedad es apenas suficiente para garantizar el reemplazo de una población cerrada (o de crecimiento natural nulo). En la figura 2, este estado está representado por la cota $K$ en el que la tasa de mortalidad es igual a la de la natalidad a un nivel de subsistencia "piso". Desde el punto de vista demográfico, una población con estas características corresponde a una población de tipo estacionario, en la que la tasa de mortalidad, $b$, es igual al recíproco del valor de la esperanza de vida al nacer.

Los dos supuestos de comportamiento se refieren, a su vez, a los componentes claves del modelo malthusiano (véase Galor y Weil 2000): la relación positiva entre las subsistencias y el crecimiento de la población,

$$
\frac{\mathrm{d} P}{\mathrm{~d} S}=\frac{r_{0} P(t)\left[1-\frac{P(t)}{K}\right]}{\gamma S}>0
$$

y la existencia de la ley de rendimientos decrecientes,

$$
f^{\prime \prime}(P)=r-\frac{2 r P(t)}{K}<0 .
$$


La manera en que estos supuestos entran en acción es a través de los cambios en el nivel de subsistencias. Para ver esto, supongamos, por ejemplo, dado un mejoramiento técnico en la dotación inicial de tierras (que es supuesta fija), la productividad del trabajo de la sociedad crece a tal grado que el nivel de subsistencias experimenta un desplazamiento de $K$ a $K_{1}$ en la figura 2. El consecuente incremento en la proporción media de subsistencias inducirá a los habitantes, según las ecuaciones de (4), a incrementar su número a un tasa $c_{2} B e^{(\gamma-r) t} / c_{1} A$ hasta el punto en que la población vuelva a ser otra vez completamente elástica con respecto al nuevo nivel de subsistencias, $K_{1}=c_{2} B / c_{1} A$. En ese intervalo, la mayor influencia de (5) en comparación con (6) garantizará que el componente $a P$ domine al componente $-b P^{2}$ en la ecuación (1).

La bonanza, dice Malthus, se perpetuará hasta que no aparezca la presión de una mayor población sobre la frontera agrícola. Una vez presente esta presión, los mecanismos comandados por la ley de rendimientos decrecientes revertirán el círculo virtuoso establecido inicialmente entre el crecimiento del ingreso y el demográfico. Los precios más altos de los alimentos ocasionados por la mayor cantidad de esfuerzo requerido para producir una unidad de producto, aunado a una baja en el salario de mercado (resultante de una oferta de trabajo incrementada por el crecimiento del periodo precedente) activarán los frenos preventivos y positivos.

Ante una disminución en el nivel promedio de subsistencias, la población reducirá drásticamente su número, no sólo por el mayor número de muertos debido a la malnutrición o a enfermedades, sino por el deseo voluntario de las familias de retrasar la edad al matrimonio y, por ende, de tener menos hijos. La combinación de (5) y (6) hará que el componente cuadrático de la primera ecuación del sistema (3) se aproxime más rápido a cero que el componente lineal y que, por tanto, la caída del crecimiento de la población sea más violenta que la de $\gamma$ en todo el trayecto de regreso al nivel de subsistencias original, $K$. Con una valor negativo mayor de $r$ que de $\gamma$, el valor del exponente de $c_{2} B e^{(\gamma-r) t} / c_{1} A$ será negativo y su límite, a medida que $t \rightarrow \infty$, tenderá a converger a cero.

La diferencia entre la ecuación (1) y el sistema (3) es que, mientras en la versión original de Verhulst no hay posibilidad de regreso al estado estacionario inicial, en el esquema malthusiano cualquier cambio positivo (cuando $P(t)<K$ ) o negativo (cuando $P(t)>K$ ) del crecimiento de la población será temporal y asintótico al nivel de $K$, sin importar las razones tecnológicas que llevaron a la elevación de $S$. La explicación formal reside en las peculiares condiciones de estabilidad y convergencia que se presentan en torno a la vecindad del punto crítico $\left(P_{0}, K\right)$ y que no son reproducibles, por ejemplo, por el punto de bifurcación $\left(P_{1}, K_{1}\right)$ de la figura 2. El análisis del polinomio característico del sistema lineal asociado a $(3), \lambda^{2}-(r+\gamma) \lambda+r \gamma=0$, revela que aún cuando los eigenvalores en la vecindad de $\left(P_{0}, K\right)$ y $\left(P_{1}, K_{1}\right)$ son reales y diferentes, en $\left(P_{1}, K_{1}\right)$ éstos son positivos, lo que significa que tanto en el sistema lineal como en el no lineal ese punto es un nodo inestable. En cambio, en la vecindad de $\left(P_{0}, K\right)$ los dos eigenvalores son negativos, lo que garantiza que las trayectorias de ambos sistemas convergerán asintóticamente a ese punto en el sentido fuerte de Liapunov, configurando así un nodo estable. 
La validez de este resultado no se alteraría ni aún con la presencia de progreso técnico exógeno ya que, en este caso, el crecimiento del ingreso sería absorbido en la misma proporción por un contingente adicional de población. El nuevo equilibrio resultante estaría situado a un nivel superior de $K$ pero con la misma pendiente, en una cantidad exactamente igual al valor del parámetro tecnológico que se tome de referencia. A esta situación, en la que el nivel de vida per capita no cambia, a pesar de la existencia de un crecimiento técnico exógeno, se le conoce como trampa malthusiana (Blanchet, 1991).

Para resumir todo lo anterior podemos decir que en este mundo malthusiano, "la fecurdidad de una familia no es una variable endógena, sino que depende, de una manera mecánica, de sus necesidades de subsistencia. Cualquier exceso de consumo por encima del nivel de subsistencia conduce a un incremento en la fecundidad, mientras que cualquier escasez no sólo reduce la fecundidad sino que incrementa la mortalidad. Este proceso incrementa o reduce el tamaño de la población dependiendo si el consumo agregado excede o no a las necesidades de susbsitencias. Es por eso que en un modelo de producción, como el Malthusiano, sin progreso técnico y rendimientos marginales decrecientes del trabajo con respecto a un factor tierra fijo, la existencia de un estado de equilibrio estacionario, en el cual el consumo es mantenido al nivel de subsistencia, es inexorable..." (Srinivasan, 1998).

\section{La adaptación del principio de población}

Lo realmente sorprendente del principio Malthusiano es que, no obstante su aparente simplicidad, su impacto en el pensaniento económico ha sido muy profundo. Prácticamente no hay un sólo modelo de crecimiento en el que no esté presente el ajuste Malthusiano de la población. ${ }^{4}$ Desde su publicación, el principio de población ha mantenido unido a la economía con la demografía y, hasta la fecha, sigue siendo el medio más socorrido por los modelos de crecimiento para justificar los peligros o bondades de una población creciente. La crítica o defensa del principio ha sido, sin duda, el principal puente de análisis de las dos disciplinas.

La tesis de Malthus tomó importancia en la economía con la formulación Ricardiana del crecimiento. David Ricardo fue el primero en incorporar el principio en un modelo dinámico en el que el crecimiento de la población y la tasa de beneficio convergían irremisiblemente a un estado estacionario. Pero, a diferencia de Malthus,Ricardo incluyó el principio no por el carácter irrefutable de

4 Samuelson (1961) utiliza, por ejemplo, la teoría malthusiana para ejemplificar el proceso de estabilidad-inestabilidad indireccional. Para tal efecto ilustra el comportamiento de la población mediante una parábola acotada en el límite inferior por el salario de subsistencia y en el límite superior por el salario de mercado, el cual es supuesto en función de los hábitos y las costumbres. Cuando la población está en su face decreciente el punto de retorno se sitúa próximo al nivel de salario de subsistencia, pero cuando la pendiente del nivel de subsistencia $f^{\prime}(S)$ hace tangencia con la curva de población, a partir de este momento, la población inicia una tendencia creciente hasta el punto en que la $f^{\prime}(S)$ hace de nuevo tangencia con la curva de población. 
sus argumentos sino para dar fundamento a su teoría del valor y, en particular, a una de las leyes más importantes de su modelo: la ley de rendimientos decrecientes. Esta ley, "hizo casi inexpugnable a la crítica el principio de población de Malthus y la sombría opinión de Ricardo sobre el desarrollo de una sociedad capitalista" (Pasinetti, 1978). La manera en que el principio se convirtió en sustrato de esa ley puede apreciarse mejor con la exposición de las ideas generales del modelo Ricardiano.

\subsection{El Modelo Ricardiano como fundamento económico del principio de Malthus.}

Ricardo desarrolló su teoría del valor para explicar cómo se determina y evoluciona la tasa de beneficio en la economía. El problema lo llevó, primero, a desarrollar un análisis que relacionara la formación del valor de cambio con la distribución de los diferentes tipos de ingresos que componen el producto excedente (beneficios, salarios y renta ) y, segundo, a demostrar que en una economía cerrada, la expansión del capital trae consigo una disminución en la productividad del trabajo en la tierra marginal; lo cual a su vez produce, suponiendo salarios constantes, un descenso en la tasa de beneficio y un aumento respectivo en la renta de la tierra.

La solución fue dada por Ricardo en dos etapas: una en el Ensayo y otra en los Principios. ${ }^{5}$ En el Ensayo, Ricardo hizo depender la tasa de beneficio de la economía de la tasa formada en la agricultura, para lo cual requirió de una teoría de la renta de la tierra. En los Principios, en cambio, invirtió los términos e hizo depender el beneficio de la economía del beneficio de la industria, alterando la versión original de la renta de la tierra. En el primer caso, la determinación del beneficio presupuso una teoría de renta de la tierra, en el segundo una teoría del valor-trabajo.

No obstante estas diferencias Ricardo dio en ambas versiones un papel primordial a la productividad del trabajo y, con ello, al principio de población de Malthus. En el Ensayo, la explicación a la baja en la tasa de beneficio $(\pi)$ es atribuida a la caída de la productividad en las tierras menos fértiles (digamos tierras de calidad $\omega$ ) que son puestas al cultivo, por efecto de una población creciente. La mayor cantidad de capital $\left(Y_{11}^{i}\right)$ requerida para producir una misma unidad de producto $\left(Y_{1}^{i}\right)$ trae como consecuencia una reducción en el beneficio de las tierras marginales y un alza en las renta $\left(\lambda_{i}\right)$ de las tierras más feraces (o de calidad $\alpha$ ), que resulta de la diferencia entre el antiguo y el nuevo tipo de beneficio en estas últimas tierras. Una vez establecida la tasa de beneficio en la tierra que no existe renta, ecuación (7), ésta se transmite, primero, hacia el resto de la agricultura mediante un ajuste de precios $\left(p_{1}\right)$, que en este caso actúan como meros numerarios, ecuación (8), y luego, por efecto de la competencia, a los demás sectores de la economía. La determinación de $\pi$

5 Los títulos completos del Ensayo (1974) y de los Principios (1977) son, respectivamente: Ensayo sobre la influencia del bajo precio del trigo sobre los beneficios de capital y Principios de Economía Política y Tributación. 
que en este caso actúan como meros numerarios, ecuación (8), y luego, por efecto de la competencia, a los demás sectores de la economía. La determinación de $\pi$ es, pues, anterior al sistema de precios y, en concreto, al precio de la renta y del salario, por lo que una teoría de la renta de la tierra requiere de una teoría del beneficio:

$$
\begin{gathered}
\pi=\frac{Y_{1}^{i}-Y_{11}^{i}}{Y_{11}^{i}} \quad(i=\alpha, \omega), \\
\left(Y_{11}^{\alpha} p_{1}\right)(1+\pi)+\lambda_{\alpha}=Y_{1}^{\alpha} p_{1}, \\
\left(Y_{11}^{\omega} p_{1}(1-\pi)=Y_{1}^{\omega} p_{1} .\right.
\end{gathered}
$$

En este esquema, el único factor que afecta el comportamiento de $\pi$ es la dificultad de producir los alimentos a causa de la omnipresente acción de la ley de rendimientos decrecientes. El razonamiento es como sigue: las alzas en el valor de cambio de los alimentos, que resultan de una disminución de la productividad del trabajo en las tierras menos fértiles, provocan subidas en los salarios nominales. Los incrementos de los salarios nominales aumentan, a su vez, en el más puro espíritu Malthusiano, el límite natural de medios de subsistencia y, en consecuencia, la tasa de crecimiento de la población. Con una población creciente, la demanda por más alimentos obliga a incorporar tierras de menor calidad y, con ello, a elevar la renta de la tierra a costa de menores beneficios. La combinación de mayores rentas y salarios conducirá al descenso secular de la tasa de beneficio de los agricultores $y$, por efectos de ajustes en los precios de los bienes-salarios regulados por la ecuación (9), también la de los industriales (quienes de esa manera se verán obligados a pagar salarios más altos), hasta el punto en que la tasa de crecimiento de $\pi$ y de la población sean asintóticas a $K$, tal como se describe en la figura 2.

Los movimientos de la población son así transmitidos por los cambios que experimenta el nivel de subsistencias. Pero a diferencia del análisis de Malthus, esos movimientos determinan y son determinados por el comportamiento de $\pi$, vía los salarios, y no sólo por la acción correctiva de los frenos preventivos y positivos o por respuestas automáticas a $S$. En el Ensayo todo aquello que afecte a $\pi$ afecta directamente al crecimiento de la población y viceversa, bajo el supuesto de que el mecanismo económico, arriba descrito, opera en ambos sentidos. La baja en los salarios reales, por ejemplo, puede hacer crecer a $\pi$ y a la población siempre y cuando la sociedad se encuentre en un estado progresivo (o cuando la tasa de crecimiento del capital sea mayor que el de la población), pero no en un estado regresivo (cuando la tasa de crecimiento del capital sea menor que la del crecimiento de la población), ya que en este caso el efecto recesivo sobre $\pi$ podría ser mayor. De igual modo, las eventuales reducciones en la tasa de crecimiento de la población podrían, en el estado estacionario, aumentar $\pi$, si la baja resultante de los salarios logra compensar los efectos negativos de la ley de rendimientos decrecientes derivados de agregar tierras menos fértiles al cultivo.

En los Principios, Ricardo le dio su forma más acabada al modelo poblacional de Malthus. Para empezar, abandonó los supuestos restrictivos del Ensayo al formular su teoría del valor en la que los valores de cambio de las 
mercancías se expresan como una razón directa de su cantidad de trabajo incorporada. ${ }^{6}$ Con esta teoría, Ricardo no sólo salvó el obstáculo relativo a la determinación de los precios de diferentes mercancías incorporadas en la canasta de bienes-salarios, sino que dio, por primera vez, una visión dinámica del comportamiento de $\pi$ y de la población. En efecto, tras la eliminación del supuesto adoptado en el Ensayo de que los insumos y el capital eran cantidades diferentes del mismo bien, Ricardo encontró en su teoría un medio general para calcular el valor de $\pi$ en la economía o en cualquier sector, cuando ésta incorporaba el cómputo de precios relativos. La ecuación (9) ilustra la formulación de $\pi$ para. el caso del sector agrícola, en donde $p_{1}$ es el precio del bien agrícola y $p_{2}$ el precio del bien industrial.

$$
\pi=\frac{Y_{1}^{i}-\left[Y_{11}^{i}+Y_{12}^{i}\left(\frac{p_{2}}{p_{1}}\right)\right]}{Y_{11}^{i}+Y_{12}^{i}\left(\frac{p_{2}}{p_{1}}\right)} .
$$

Contrario al resultado obtenido en el Ensayo, esta ecuación ejemplifica el hecho de que los precios preceden el cálculo de $\pi$. De otra manera, habría indeterminación en éste y en los otros dos componentes (salarios y rentas) del producto total de la economía. Sin un sistema de precios no habría, según Ricardo, explicación posible de la evolución de la economía. Por eso, es que en su nueva versión de la renta de la tierra, es el alza de los precios agrícolas, debido a la caída de la productividad del trabajo, $f^{\prime}\left(P_{1}\right)$, lo que provoca la formación y el aumento de renta en las tierras más feraces; o el cambio en los precios de los bienes-salarios lo que fija el nivel del salario de mercado $S_{1},{ }^{7}$

Con un sistema de precios basados en la teoría del valor, el problema de la fijación de $\pi$ en toda la economía se vuelve entonces un asunto fácil de comprender al observar las condiciones de la industria. $\mathrm{Y}$ es que, debido a que estas condiciones permanecen, por hipótesis, constantes, el alza de los precios relativos de los bienes-salarios de la agricultura con relación a los de los productos industriales hará disminuir la tasa de beneficio industrial. La acción posterior de la competencia provocará que ésta tasa se imponga al resto del capital invertido en la agricultura (Benetti, 1972).

No obstante las diferencias en la determinación y fijación de $\pi$ entre el Ensayo y los Principios, la conclusión acerca de la evolución de la economía y de la población hacia el estado estacionario es mas o menos parecida en

6 Entre los supuestos restrictivos cabe destacar el hecho de que el producto y el capital son considerados como cantidades diferentes del mismo insumo (trigo) y que todo el capital es circulante; por lo que no quedan residuos susceptibles de utilización durante el periodo de producción siguiente.

7 Esta conclusión es contraria a la obtenida en el Ensayo en donde la formación de la renta es la que provoca el alza de precio del bien agrícola. En la nueva teoría de la renta se puede presentar el caso de que aún con tasas de beneficio iguales en tierras de distinta calidad, exista renta en las tierras de mayor calidad a causa del alza en el precio del bien agrícola. Una situación impensable en el Ensayo. 
ambas versiones. La ecuación (10) sintetiza la posición que Ricardo mantuvo al respecto en los Principios, al mostrar el comportamiento dinámico de $\pi$ ante cambios en $K$, que aquí es tomado como capital circulante o capital dedicado al pago de salarios. De acuerdo con esta ecuación, la tasa de cambio de $K$ con respecto a $\pi$ es directamente proporcional a $f^{\prime}(P)$ y a $\mathrm{d} P / \mathrm{d} K$, e inversamente proporcional a $f^{\prime \prime}(P)$ y al salario natural $S_{0}$. La explicación se encuentra en el signo negativo que acompaña a los términos en que $f^{\prime}(P)$ aparece como denominador (pues, por hipótesis, $f^{\prime}(P)=F\left(S_{1}-S_{0}\right)>0$ si la economía no se encuentra en un estado estacionario y $\left.f^{\prime \prime}(P)<0\right)$ y en la relación positiva que, por la ecuación (5), mantiene la expresión $\mathrm{d} P / \mathrm{d} K$, donde $K$ sustituye en este caso a $S$.

$$
\frac{\mathrm{d} \pi}{\mathrm{d} K}=\frac{1}{f^{\prime}(P)}\left[\frac{f^{\prime}(P)}{S_{0}}-1+K \frac{f^{\prime \prime}(P)}{f^{\prime}(P)} \cdot \frac{\mathrm{d} P}{\mathrm{~d} K}\right] .
$$

Los efectos de la acumulación de $K$ sobre $\pi$ serán, entonces, positivos si el crecimiento de la productividad del trabajo es capaz de compensar los efectos negativos de la ley de rendimientos decrecientes y del crecimiento desmesurado de la población. En general, d $\pi / \mathrm{d} K$ será positivo al inicio del proceso de acumulación si $K=0$ y la productividad del trabajo es mayor al salario natural, y durante el proceso de acumulación si la acción de los rendimientos decrecientes no son mayores que la productividad del trabajo. En el estado estacionario, esto es cuando $f^{\prime}(P)=S_{0}$, la ecuación (10) siempre será negativa a causa de los rendimientos decrecientes (véase Pasinetti, 1978). En cualquiera de los dos casos, el papel del principio de población de Malthus es de primera importancia pues, además, de que condiciona el movimiento de cada una de las variables del lado derecho de la ecuación (10), de la manera arriba descrita, asegura que la convergencia hacia el estado estacionario del sistema (representado por el par de soluciones linealmente independientes $S_{1}=S_{0}$ y $\left.f^{\prime}(P)=S_{0}\right)$ sea estable.

Para tener una idea más clara sobre este último punto, hay que tener en mente que Ricardo distingue entre ingreso neto $I_{n}$, o fondo del cual dependen los terratenientes y capitalistas, e ingreso bruto $I_{b}$, o fondo del cual depende la reproducción de la clase trabajadora. La suma de los dos da, obviamente, el ingreso total $I_{T}$ producido por la economía en un año. La evolución de cada uno de estos ingresos es una función directa de la aplicación de la tecnología en el proceso de acumulación de capital. En concreto, Ricardo sostiene que mientras $I_{n}$ crece regularmente con el uso de la tecnología, el valor de $I_{b}$ tiende a decrecer hasta hacerse asintótico al nivel del salario natural, $S_{0}$. El crecimiento de la población trabajadora es entonces diferenciado del de las otras dos clases minoritarias, tal como se expresa en el sistema (11).

$$
\begin{aligned}
\frac{\mathrm{d} P_{\tau}}{\mathrm{d} t} & =r_{0} P_{\tau}(t)\left[1-\frac{P_{\tau}(t)}{K}\right], \\
\frac{\mathrm{d} I_{b}}{\mathrm{~d} t} & =\Theta\left(I_{T}-I_{n}\right) I_{T}, \\
I_{b_{0}} & =S_{0}=K,
\end{aligned}
$$

donde: $\Theta$ es el parámetro tecnológico y $P_{\tau}$ es la población trabajadora. 
Las condiciones de estabilidad y convergencia de este sistema son iguales a las presentadas por la curva solución del sistema (3), excepto por el hecho de que las cotas superior e inferior de la nueva curva solución para $P_{\tau}$ están definidas por el máximo $I_{b_{1}}$ y el mínimo $I_{b_{0}}$. La amplitud de la diferencia entre las cotas de la figura (3) es una función de la naturaleza de $\Theta$, ya que entre más intensivo en capital fijo (o capital dedicado a la construcción de maquinaria) sea el uso de la tecnología en el proceso de acumulación con relación al capital circulante, menor será el techo máximo del crecimiento de la población y más rápido será su convergencia al nivel $I_{b_{0}}$ o viceversa.

Figura 3. Equilibrio Demográfico Ricardiano.

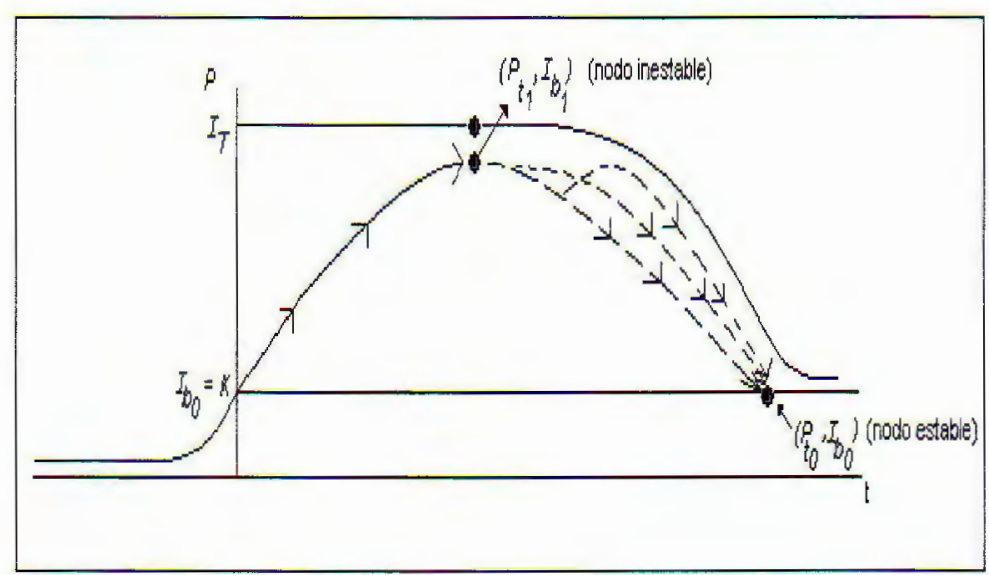

\section{Conclusiones}

El presente trabajo muestra que el principio de población de Malthus es esencial en el modelo clásico de crecimiento para explicar la estabilidad y convergencia hacia el estado estacionario. Las modificaciones introducidas en la ecuación original de Verhulst hacen explícitas las diferencias introducidas por Ricardo al principio de población. La sustitución del concepto Malthusiano de subsistencias por el de ingreso bruto; así como el de la población como agente depredador por el de la población como niveladora del fondo de salarios, permitieron a Ricardo dar al principio un contenido económico que no tenía con Malthus. Para empezar, lo despojó de su relación meramente biológica y lo incluyó en el ámbito de la reproducción social. Ahora, la población crecería o decrecería por el efecto de mecanismos económicos comandados por los vaivenes de la tasa de beneficio y no sólo por la acción de los niveles de subsistencias. Además, los mecanismos de transmisión entre las variables económicas y demográficas podían ser alterados por la naturaleza de la tecnología así como por el estado de la acumulación (esto es, dependiendo si este estado fuera regresivo o progresivo) y no únicamente por los frenos preventivos o positivos.

No obstante, estas diferencias la importancia del principio en el modelo clásico es tal que sin su inclusión quedaría inexplicada la ley de rendimientos decrecientes y, más aún, la tendencia convergente y estable al estado estacionario. 
La adaptación de este principio por Ricardo sentó, de hecho, las premisas para formular modelos de crecimiento más sofisticados en los cuales las variables económicas y demográficas deberían crecer a determinadas tasas al momento de alcanzar un estado estable (modelos neoclásicos), inestable (Keynesianos) o estacionario (clásico).

\section{Bibliografía}

Benetti, C. (1972). Valor y Distribución. Maspero, Madrid.

Birsdall, N. (1993). Economic approaches to population growth. In Chenery, H., and T. N. Srinivasan, Handbook of Development Economics, $(1,12)$ Elsevier, Amsterdam.

Blanchet, D. (1991) Modélisation Démo - Économique. Conséquences économiques des evolutions démographiques, Institut National dÉtudes Démographiques, Paris.

Eltis, W. (2000). The Classical Theory of Economic Growth. Palgrave, New York.

Galor, O., and N. D. Weil (2000). Population, technology and growth: from malthusian stagnation to the demographic transition and beyond. The American Economic Review 90(4), pp. 806-828.

Keifitz, N. (1983). The evolution of Malthus's thought: Malthus as a demographer. In J. Dupaquier. Malthus Past and Present. Academic Press, London.

Le Bras, H. (1983). Malthus and the two mortalities. In J. Dupaquier. Malthus, Past and Present. Academic Press, London.

Malthus, T. R. (1977). Ensayo sobre el Principio de la Población. FCE, México.

Pasinetti, L. L. (1978). Crecimiento Económico y Distribición de la Renta. Alianza Universidad, Madrid.

Ramírez, José C. y J. Morelos (2002). El concepto de población en los modelos de crecimiento económico. Trimestre Económico, 69(2), pp. 145-190.

Ricardo, D. (1974). Ensayo sobre la influencia del bajo precio del trigo sobre los bebeficios del capital en Napoleón, C. Fisiocracia, Smith, Ricardo y Marx. Oikos-Tau, Barcelona.

Ricardo, D. (1977). Principios de Economía Política y Tributación. FCE, México.

Samuelson, P. (1961). Foundations of Economic Analysis. Harvard University Press, Cambridge, Mass.

Srinivasan, T. N. (1998). Introduction to part 3. In Chenery, H., and T. N. Srinivasan, Handbook of Development Economics, 1, Elsevier, Amsterdam.

Winch, D. (1997). Malthus . In R. Donald, D. Winch, and Skidelsky. Three Great Economists. Oxford University Press. 